\title{
LA PALMICULTURA COLOMBIANA: SOSTENIBILIDAD ECONÓMICA, SOCIAL Y AMBIENTAL
}

\author{
Por: Aylin Patricia Pertuz Martínez ${ }^{1}$ - Álvaro Enrique Santamaría Escobar ${ }^{2}$
}

\section{RESUMEN}

El cultivo de palma de aceite en Colombia ha tenido una expansión importante, producto de políticas gubernamentales que lo consideran un factor de desarrollo para la economía agraria y del país. Con un recorrido de más de cincuenta años, cuenta actualmente con una producción en cuatro zonas reconocidas, en donde la mayor parte de los productores están asociados a Fedepalma. Un aspecto que genera debate en el país es la sostenibilidad del sector en sus componentes económico, social y ambiental. El objetivo de esta revisión es determinar si en el país se ha desarrollado una palmicultura sostenible. Se concluye que este sector agrario es sostenible en lo económico para el gran productor, pero no es sostenible en lo social y ambiental, aunque hay avances puesto que se aspira a contar con certificaciones internacionales. Por lo general, el sector es considerado sostenible por parte de las entidades gubernamentales y del propio sector, salvo excepciones.

Palabras clave: palmicultura, sostenibilidad, Colombia.

Clasificación JEL: Q01.

1. Administradora de Empresas, Magister en Administración de Empresas, Especialista en Gestión Pública; Universidad de Sucre; aylin.pertuz@unisucre.edu.co.

2. Ingeniero Industrial, Magister en Administración de Empresas, Magister en Educación, Especialista en gerencia de la Producción y la calidad: Universidad de Sucre; alvaro.santamaria@unisucre. edu.co

Fecha de recepción: 29 de noviembre de 2013 - Fecha de aprobación definitiva: 25 de mayo de 2014 
COLOMBIAN OIL PALM CULTIVATION CULTURE, ECONOMIC, SOCIAL, AND ENVIRONMENTAL SUSTAINABILITY

\author{
By: Aylin Patricia Pertuz Martínez - Álvaro Enrique Santamaría Escobar
}

\begin{abstract}
ABSTRAC
The oil palm cultivation in Colombia has had an important expansion; it has been the product of government policies that consider it a development factor for the agricultural economics and the economy of the country. After fifty years, it has a production in four known areas where most of the producers are associated to Fedepalma. An aspect that generates debate in the country is the sustainability of the sector in its environmental, economic and social components. The main purpose of this revision is to determine if in the country a sustainable palm oil cultivation culture has been developed. It is concluded that this agricultural sector is economically sustainable for the large producer, but it is not sustainable in the environmental and social aspects although there are advances due to the fact that it will become internationally certified. In general terms, the government entities and the sector itself consider it sustainable, considering some exceptions.
\end{abstract}

Key words: oil palm cultivation culture, sustainability, Colombia.

JEL Classification: Q01. 


\section{INTRODUCCIÓN}

El presente artículo tiene como propósito exponer la situación del cultivo de palma de aceite (palmicultura) en Colombia, en relación con el concepto de sostenibilidad, para lo cual se realizó una revisión de la literatura correspondiente al tema en los últimos trece años, comprendiendo artículos o documentos de organismos ambientales, entidades oficiales y privadas, libros, documentos de instituciones reconocidas en dicho ámbito, así como trabajos de grado de maestría y tesis doctorales. Su carácter es analítico interpretativo. El objetivo de la revisión es determinar si se ha desarrollado en Colombia una palmicultura sostenible.

Presenta seis secciones en las que se expone inicialmente una breve situación del cultivo de palma de aceite en el país, para luego tratar el concepto de sostenibilidad y mostrar al sector en los distintos tópicos de la misma. Por último, se exponen las conclusiones.

\section{LA PALMICULTURA EN COLOMBIA}

El cultivo comercial de palma de aceite (Elaeis guineensis y Eleais oleífera) inicia en Colombia hacia el año 1945, estando a la fecha en constante expansión. Para la década de 1960 existen unas 18.000 has en producción (García et al, 2013:79,80), y décadas después, en el año 2012, el área sembrada es de 452.435 has con un crecimiento de 5.9\% respecto al año anterior (Fedepalma, 2012: 37). Colombia se ubica en el año 2010 como el primer productor de América Latina y el quinto del mundo con un 1.6\% de la producción mundial (Superintendencia de Industria y Comercio, 2010: 3).

El cultivo de palma de aceite se promueve a partir del gobierno del presidente Andrés Pastrana (1998 - 2002) como uno de los ejes de desarrollo del país. Los derivados del fruto se destinan a la demanda nacional de grasas y aceites, así como a las exportaciones. Con los gobiernos de Álvaro Uribe (2002 - 2010) se define una nueva política y estrategia de desarrollo, en este caso la producción de biocombustibles (Konrad Adenauer Stiftung, 2008: 2), teniendo en cuenta que los rendimientos más altos de conversión en el país se dan con esta palma (Acevedo, 2012:100). En el gobierno actual de Juan Manuel Santos, el sector hace parte del plan "Ola Agro”.

De acuerdo con el Ministerio de Medio Ambiente Vivienda y Desarrollo Territorial y Fedepalma (2011:6), del cultivo de palma existen unas 365.000 hectáreas distribuidas en 16 departamentos y 106 municipios, los cuales conforman cuatro zonas palmeras: zona Norte (Norte del Cesar, Atlántico, Guajira, Norte de Bolívar, Córdoba, Urabá Antioqueño y Sucre), Zona Central (Santander, Norte de Santander, Sur del César, Sur de Bolívar), Zona Oriental (Meta, Cundinamarca, Casanare, Caquetá), Zona Occidental (Nariño, Cauca).

Lideran la cadena del sector las organizaciones: Fedepalma, Cenipalma, Acepalma, Fondo de Fomento Palmero y Fondo de Estabilización de Precios (Mosquera et al, 2009: 13, 41, 57). 


\section{EL CONCEPTO DE SOSTENIBILIDAD}

El informe Brundtland (1987:59), denominado "Nuestro futuro común”, indica que "el desarrollo sustentable (duradero) es el desarrollo que satisface las necesidades de la generación presente sin comprometer la capacidad de las generaciones futuras para satisfacer sus propias necesidades”. Tal como expresa Rojas (2003: 10, 231), el desarrollo sustentable o sostenible, contiene tres elementos sustanciales para el desarrollo: lo social, lo ambiental y lo económico. Lo social hace referencia a la equidad y un mejor entendimiento de "la interdependencia de las comunidades humanas". Lo ambiental, se relaciona con el uso del "capital ecológico preservando la productividad del entorno a largo plazo”. Lo económico, se dirige al crecimiento económico sostenido, que genere el incremento del ingreso y no aplique políticas de corto plazo que conduzcan a un empobrecimiento en el largo plazo.

Para la CEPAL (199:24-25), citado por Nieves (1998:10), la sustentabilidad del desarrollo requiere un equilibrio dinámico entre todas las formas de capital o acervos que participan en el esfuerzo del desarrollo económico y social de los países, de tal modo que la tasa de uso resultante de cada forma de capital no exceda su propia tasa de reproducción [...] Entre las formas de capital más importantes cabe destacar el capital humano (en que las personas también representan el sujeto del desarrollo), el capital natural, el acervo institucional (los sistemas de decisiones), el acervo cultural, el capital físico (infraestructura, maquinarias y equipos) y el financiero".

\section{LA PALMICULTURA COLOMBIANA Y SU SOSTENIBILIDAD}

La palmicultura, considerada en los últimos gobiernos nacionales como una "locomotora del desarrollo" para el país, es una actividad que ha avanzado con buena dinámica en las dos últimas décadas, pero con algunas visiones encontradas acerca de su sostenibilidad. De acuerdo con el Ministerio de Ambiente, Vivienda y Desarrollo Territorial (hoy Ministerio de Medio Ambiente y Desarrollo Sostenible) y Fedepalma (2011: 5), el sector viene realizando desde 1997 ingentes esfuerzos con diversas instituciones públicas para adelantar acciones que permitan al sector una producción limpia, reducir niveles de contaminación, reducir riesgos para el medio ambiente y la población, así como proteger el capital natural del planeta. Asimismo, desde el año 2008, Fedepalma está realizando acciones para ser miembro del proyecto RSPO, Mesa Redonda de Aceite de Palma Sostenible (por sus siglas en inglés), creado en Suiza en el año 2004 debido a los problemas ambientales y sociales generados en países como Malasia e Indonesia. La RSPO posee criterios y principios que deben acatarse para certificar los productos que llegan al consumidor (Ocampo, 2009: 178).

Con base en esto: ¿cómo se ha desarrollado el cultivo de palma de aceite en los últimos trece (13) años desde el marco de la sostenibilidad?

\section{SOSTENIBILIDAD ECONÓMICA}

Las grandes inversiones en los cultivos de palma tienen que estar soportadas en diversas variables como la capacidad económica para establecer la plantación y 
esperar tres (3) o cuatro (4) años para obtener su fruto; la posibilidad de sobrevivir a las fluctuaciones del mercado; la facilidad para obtener préstamos bancarios; la posibilidad de integrar las plantaciones con la industria extractora y refinadora...; el poder político para influenciar las decisiones gubernamentales; y la posibilidad de invertir en infraestructura para la distribución (Mingorance et al, 2004: 24, 25). El cultivo requiere de un alto nivel de inversión para su establecimiento, debido a la preparación y mantenimiento del terreno en los primeros años improductivos, y al montaje de la infraestructura necesaria, desde la explotación hasta la extracción del aceite (Martínez et al, 2005: 20).

Empero, es un cultivo altamente productivo por hectárea/año. En el mercado mundial su precio es bajo y estable comparado con otros aceites vegetales, haciéndolo altamente competitivo en este mercado y viable económicamente para el biodiesel (Avellaneda, 2010: 6).

Entre los años 1996 y 2006 la participación promedio de valor agregado de la producción de aceite de palma en el PIB colombiano es de 1.6\% con un crecimiento anual de 3.5\%. En las exportaciones, los principales beneficios económicos se dan entre los comercializadores, transformadores y grandes productores, dejando por fuera a los pequeños cultivadores (Pérez, 2010: 5). Las perspectivas al 2020 pretenden multiplicar la producción a 3.5 millones, e incrementar la productividad a 5.5 toneladas, con una tasa de crecimiento del $8 \%$ anual y un crecimiento de exportaciones de un 78\% (Sánchez, 2009: 3).

Los mayores ingresos se dan a partir del año seis (6) y para optimizar los costos fijos y ser competitivo, según Fedepalma, se requieren 5.000 has. en promedio (citado por Ocampo, 2009: 176,177). Para el año 2005 el costo de producción es de 330 US $\$ / t$, en tanto que el de Indonesia apenas supera los 200 US\$/t y el de Malasia es de 280 US\$/t. En el año 2007, el costo de producción aumenta a 340 US\$/, a diferencia de Indonesia y Malasia que se reduce a 154 US\$/t y 216 US\$/t, respectivamente (Infante; Tobón, 2010: 46).

En cuanto al rendimiento del aceite de palma, este presenta una reducción de 8.3\% en el año 2012, al pasar de 3.54 a 3.25 ton/ha. La productividad en Malasia es $18 \%$ superior, por lo cual se deben hacer esfuerzos para cerrar esta brecha de productividad. En el año 2012 se presenta una caída abrupta de los precios producto de la caída de los precios internacionales, la desgravación de los aceites vegetales importados (Acuerdo de Libre Comercio) y la revaluación del peso colombiano frente al dólar (Fedepalma, 2012: 38,42). Es de anotar que en fechas recientes, desde el 2007 se reducen los rendimientos por hectárea debido a enfermedades y plagas (Silva; Martínez, 2009: 98).

Ahora bien, la producción de aceite de palma en Colombia no es competitiva si se compara con los países líderes que presentan costos de producción más bajos; pero los mayores costos de producción se trasladan al consumidor final "reduciendo su bienestar" (Cortés et al, 2012: 95). Según Finagro (2012), aunque el rendimiento promedio de Colombia en el año 2009 es de 3.4 ton/ha, el cual es inferior a Malasia e Indonesia, es superior al promedio mundial de 2.99 ton/ha (citado por García; Calderón, 2012: 75) 
Para Indupalma S.A., organización perteneciente a Fedepalma, el ejercicio económico desde al año 2006 es positivo, siendo sus mayores impactos la contratación laboral, el apoyo a proveedores, y a las Unidades Autónomas Empresariales, incluidas en las Cooperativas Asociativas de Trabajo (Indupalma, 2011: 38,39).

Según Fedepalma, la evaluación a 23 alianzas que conforman al modelo de Alianzas Productivas Estratégicas (relaciones formales entre productores del sector), muestra que hay una mayor facilidad para la financiación, asistencia técnica y mayor formalización de relaciones con el Estado. Factores por mejorar: el estado sanitario de los cultivos, el orden público, créditos bancarios entregados inoportunamente y los cambios repentinos de los precios internacionales. Según la entidad, tanto la empresa como los productores ganan. (Castiblanco; Hortúa, 2012: 17). Vidal y Villaroel (2011: 148-157), en estudio realizado en María La Baja (Bolívar), consideran que el sistema de alianza estratégica entre pequeños productores de palma de aceite y los grandes productores y/o extractores genera beneficios a ambas partes y permite el sostenimiento económico de todos los involucrados.

El cultivo de palma aceitera es exitoso en el país en la última década debido a una proyección estratégica internacional, que tiene como base un "sistema organizacional especializado", unido a políticas del Estado que le han sido favorables (López, 2000: 131). En este sentido, el cultivo ha recibido grandes recursos por parte del Ministerio de Agricultura, que expresa que el 49\% de la propiedad es de los mismos agricultores. En la región de los Montes de María, los campesinos señalan que las tierras, en su gran mayoría, pertenecen a una sola familia (Preti et al, 2010: 8).

La sostenibilidad económica del cultivo de palma de aceite tiene visiones encontradas en lo que respecta al pequeño y mediano productor, aunque la mayoría de autores considera que los pequeños productores no disfrutan de los beneficios económicos. En el sector, los grandes empresarios inducen a los campesinos a asociarse en proyectos de monocultivo gracias al endeudamiento. En realidad, los primeros sólo desean obtener materia prima sin ninguna vinculación laboral (Carrere, 2001:53). La apuesta exportadora del gobierno, en la que se encuentra la palma de aceite, implica convertir a los campesinos en empresarios prósperos, pero esto se evapora porque los pequeños campesinos serán desplazados a los cinturones de miseria de las ciudades, y algunos pocos solo serán mano asalariada de nuevas empresas exportadoras con pésimos sistemas de contratación (Tobasura, 2009: 31). Un punto de vista diferente es el que considera que la actividad palmera aumenta el empleo y le da estabilidad económica a los palmeros. En regiones como Tumaco, es percibida, entre otros aspectos, como una fuente de ingresos para la mano de obra no calificada (Perfetti et al, 2003:112). En la experiencia de Sabana de Torres (Santander), se considera que el modelo asociativo es válido para los pequeños productores, aunque, para ello, es necesario el acompañamiento de Ongs, al igual que la capacitación en aspectos asociativos (Baldovino, 2011:94).

\section{SOSTENIBILIDAD SOCIAL}

Las empresas palmeras en el país han sido objeto de debate en lo relacionado con el impacto social de su actividad. Así por ejemplo, en el Urabá chocoano y 
antioqueño las empresas palmicultoras han adquirido más de 15.000 has de tierra, siendo investigados algunos empresarios por desplazamiento y cercanía a grupos irregulares (Rivas et al, 2011: 22). Respecto a estos grupos, en el Pacífico se presentan dos periodos relacionados: antes de 1991, la guerrilla realizaba acciones sobre los negocios locales y posteriormente, los paramilitares empezaron a consolidar su expansión territorial con los cultivos de palma (García, 2011: 276).

En el Magdalena Medio también se ha presentado una expansión del cultivo de palma aceitera que ha concordado con la expansión de los grupos irregulares. A pesar del discurso de Fedepalma, manifestando que gracias a Indupalma S.A. se ha logrado estabilidad económica y procesos de paz, la realidad es diferente. De acuerdo con Hurtado y Hernández (2010: 141), la mejoría en seguridad que ha tenido la región en los últimos años no debe verse como ausencia de conflicto, sino como la supremacía de un grupo sobre otro. En el Municipio de San Alberto y San Martín (Cesar), el desplazamiento forzado da muestra del conflicto presente en la zona que involucra también a ciertos políticos.

Entre 1995 a 2005 son desplazados 20.150 hogares de 30 municipios del Magdalena Medio (Fian Internacional, 2009: 21). También es conocido el caso de conflictos de tierras en el Sur de Bolívar en el que fueron desalojadas 123 familias del territorio en que estaban asentadas (Hurtado; Pereira, 2011: 93). En el año 2004, 17.000 has de tierras (Carimagua, predio en el Departamento del Meta) habían sido destinadas a desplazados (campesinos expulsados de sus tierras), pero estas fueron otorgadas a grandes empresarios de la palma aduciendo que las tierras eran ácidas y los desplazados no tenían experiencia para ese manejo, lo cual es una forma de confundir a la opinión pública (Castrillón, 2008: 259.)

Grandes afectados por el monocultivo de palma aceitera son los campesinos sin tierra ubicados en áreas de propietarios a través de la aparcería, así como en las sabanas y playones comunales (baldíos) en donde radican sus cultivos. Perder estas posibilidades los ha dejado sin medios para ganarse la vida, colocándolos en riesgo alimentario, como acontece en el municipio de Simití al Sur de Bolívar (Álvarez, 2009: 90).

En cuanto al empleo y las relaciones laborales, la palmicultura no es una gran generadora de empleo, puesto que por cada 100 has se emplean sólo 27 trabajadores. Es común el sistema de flexibilización laboral en forma de subcontratación, lo cual ha permitido la competitividad del sector para el empresario. Así, con la creación de las Cooperativas Asociativas de Trabajo se han disminuido los costos, aumentado la rentabilidad para el gran empresario, y se ha limitado o impedido la creación de sindicatos, tanto en el Magdalena Medio como en el Meta. Las condiciones de trabajo tampoco son las más adecuadas (Ramírez, 2012: 45). En Santander, en algunas organizaciones palmeras, los sindicatos se debilitan mediante planes de retiro voluntario con indemnizaciones más altas (Carrere, 2006: 41)

En cuanto a la estabilidad laboral y la seguridad social, en Cumaral, Meta, existe una estabilidad laboral, aunque los sueldos no son más representativos que otras actividades agrícolas, existiendo diferencias en las condiciones laborales entre las distintas empresas localizadas en la zona. Los trabajadores se encuentran afiliados al sistema de seguridad social del país, pero el acceso al sistema depende del cargo 
que tenga el trabajador; y las empresas no tienen programas de salud que se extienda a las familias de sus trabajadores (La Rotta, 2010: 93-94-95,111).

En la zona bananera del Magdalena, la transición de la explotación de banano a la de palma hizo disminuir la mano de obra y la seguridad alimentaria, produciendo desplazamiento, peor aún en medio de un conflicto, y al igual que el Pacifico con adquisición de tierras en forma legal e ilegal (Goebertus, 2008: 153,156,162).

Los que consideran que existe sostenibilidad social, en el sector, exponen como un negocio inclusivo a Indupalma S.A., puesto que los campesinos que antes eran jornaleros ahora son propietarios, gracias a que la empresa logró que estas personas obtuvieran financiación por doce (12) años con cuatro (4) años de gracia, para la compra de la tierra y el establecimiento del cultivo. Hay treinta (30) cooperativas de trabajo con 1.300 familias, de las cuales 500 son dueñas de sus tierras (Londoño; Pineda, 2008: 25). Gracias al modelo de Indupalma S.A., los campesinos son gerentes de cooperativas, han mejorado su productividad y se sienten orgullosos en el Caribe colombiano de vivir esta experiencia. Este cultivo permitirá el desarrollo económico y social del Caribe (Aguilera, 2002: 3, 35, 42).

De acuerdo con un estudio de Olivera et al (2011: 88), las condiciones de empleo de los trabajadores palmicultores son mucho mejores que las de los no palmeros; hay un gran cumplimiento de la normatividad laboral, presentan mejores ingresos y tienen más posibilidades de acceder a seguridad en salud y pensiones que los no palmeros.

Respecto al proceso seguido en Colombia para ser miembro de la RSPO, parece más bien un proceso para limpiar la imagen negativa del sector palmicultor. Oxfam Novib se pregunta si el proceso es factible en el país por las siguientes razones: baja participación en el proceso; Fedepalma tiene mucho poder en el gobierno, agudizado por la puerta giratoria (miembros del sector privado ocupan cargos públicos y luego vuelven al sector privado); la élite del país está ligada a la tierra; $\mathrm{y}$, el sector palmicultor observa quienes tienen ideología de izquierda, como radicales y terroristas; al contrario, al sector se le ve como violador de los derechos humanos. De todos modos, el proceso puede conducir a algún cambio (Seebolt; Salinas, 2010: 13, 33, 35, 36).

\section{SOSTENIBILIDAD AMBIENTAL}

Colombia, es la primera potencia mundial en biodiversidad relativa, pero también es la campeona en depredación ambiental, debido a ciertos factores entre los cuales se encuentran algunas actividades agrícolas (Carvajal; De Francesco, 2012: 469). Desafortunadamente, los aspectos ambientales no tienden a ser la prioridad en las políticas gubernamentales que impulsan la utilización de los biocombustibles. Los cultivos que se utilizan para este fin generan la mayor parte de los impactos ambientales negativos debido a la utilización intensiva de agroquímicos, degradación de suelos y sobrexplotación de recursos hídricos (Coviello et al, 2008: 103), lo que parece validar el título señalado acerca de Colombia.

En el Departamento del Cesar, para el año 2003 existían 29.536 has de cultivo ubicadas en su mayoría en el sur del departamento. Esto ha generado gran contro- 
versia entre los campesinos quienes han manifestado que la planta afecta la tierra debido a la gran cantidad de nutrientes que absorbe y por la forma como cambia al suelo al dejarlo sin posibilidades para otros cultivos en el futuro. También existe controversia por la utilización de bosques protegidos, y el uso y disposición indiscriminada de agroquímicos es una amenaza para el área de acuíferos (Preti et al, 2010b: 23-24). En la región del Magdalena Medio, este cultivo ha venido ocupando espacios de zonas boscosas y ciénagas de ríos, haciendo desaparecer algunos cuerpos de agua como caños (Soler; León, 2009: 20,22-23).

En este departamento, la palmicultura no respeta las zonas de retiro a las corrientes hídricas y nacimientos donde se localizan, lo cual puede generar impactos negativos en la oferta de agua de esta zona, que en su mayoría son zonas secas, con cultivos que deben recibir agua de sistemas de irrigación (López, 2010:65-66).

En la Orinoquía no existe armonía entre lo expresado por Fedepalma y sus asociados, puesto que la generalización del drenaje del suelo puede constituirse en una amenaza para la estabilidad de los ecosistemas regionales, en especial porque hay ausencia de ordenamiento territorial efectivo (Rodríguez et al, 2009: 37-38).

Un caso de mucho cuidado se presenta en el Departamento del Chocó, debido a la invasión de territorios en zonas de titulación declaradas reserva forestal, pero las instituciones ambientales no han ejercido ninguna labor al respecto; además, los cultivos están amparados por el Plan Colombia. Según el gobierno, los cultivos no afectan los bosques vírgenes del país, pero esta afectación se ha dado en el denominado Chocó Biográfico (Seebolt; Salinas, 2010:22,31).

En el Bajo Atrato, se observa la reducción de la disponibilidad del recurso hídrico para las comunidades de la localidad, hay una reducción en el nivel freático de los suelos intervenidos, así como una afectación de la "red de drenaje natural en dichos suelos" (Cuesta, 2009:41).

Respecto a la utilización de las fincas cultivadas con palma, los empresarios del sector afirman que el $82.5 \%$ eran utilizadas antes para la ganadería y agricultura y un $17.5 \%$ eran ecosistemas naturales. A pesar de esta afirmación se presentan controversias por las plantaciones en el Municipio de Tumaco, Nariño. El subsector ha dado pasos importantes en lo ambiental, pero no todas las fincas cumplen con este compromiso (Rodríguez; Van Hoff, 2003: 3).

Colombia es un país de una gran biodiversidad, la cual corre un gran riesgo dado la presión sobre su "capital natural" ejercido por este tipo de cultivos. Es de anotar, que en Malasia e Indonesia desparecieron más de cinco millones de hectáreas de bosque tropical, debido al cultivo de palma de aceite (Konrad Adenauer Stiftung, 2008: 2), con la consecuente desaparición o disminución de especies animales.

Defensores del cultivo sostienen que el cultivo de palma no es un cultivo tan depredador como la ganadería. En el caso del llano colombiano, las áreas que antes se usaban para la ganadería, ahora son utilizadas para el cultivo de palma aceitera, lo cual es una consecuencia ambiental positiva. En términos de captura de $\mathrm{CO}_{2}$, es menos dañina la tala de árboles para palma que para la ganadería (Ochoa; Chávez, 2011: 80). De acuerdo con Fedepalma, existe un gran interés en el gremio para preservar la biodiversidad del país (Fedepalma, 2012: 10) y de acuerdo con 
los discursos de Álvaro Uribe, en relación con los cultivos de palma de aceite y biocombustibles, estos no afectan a la selva ni los bosques de la Amazonía colombiana (Wilches, 2011: 71).

\section{CONCLUSIONES}

La revisión bibliográfica muestra que la palmicultura es sostenible económicamente para el gran empresario, dada la productividad y rentabilidad del cultivo, aunque no es cercano a lo visto en los países líderes. Ahora bien, el sector ha presentado períodos complicados por factores nacionales e internacionales. Respecto a los pequeños productores las publicaciones del gremio defienden el modelo de "alianzas" y sostiene la sostenibilidad del pequeño y mediano productor. Para otros autores, estos últimos no tienen sostenibilidad económica, más bien hacen parte de una estructura para la disminución de costos de la gran empresa.

La palmicultura no es sostenible social ni ambientalmente, puesto que en muchas partes del territorio ha estado ligada a las violaciones de los Derechos Humanos. Asimismo, los grandes monocultivos de palma de aceite para los biocombustibles son una amenaza para la seguridad alimentaria y lesionan la biodiversidad. Por lo general, son los gremios y las entidades gubernamentales quienes afirman tener esta sostenibilidad con reservas sobre ciertas zonas en el aspecto ambiental.

\section{REFERENCIAS}

ACEVEDO, Paola (2012). Herramientas de análisis de alternativas de producción incorporando el ACV "cuna a cuna" a los métodos tradicionales. Comparación de biodiesel de palma e higuerilla. Universidad Industrial de Santander, Escuela de Ingeniería Química, Tesis doctoral. Bucaramanga, Colombia, $223 p$. (En línea) Disponible en http://repositorio. uis.edu.co/jspui/bitstream/123456789/7414/2/145395.pdf (consultado sep. 15-13).

AGUILERA, María (2002). Palma africana en la costa Caribe: un semillero de empresas solidarias. Documentos de Trabajo sobre Economía Regional, Banco de la República, Centro de Estudios Económicos y Regionales, No 30, 55p. (En Línea) Disponible en http://www.banrep.gov.co/sites/default/files/ publicaciones/archivos/DTSER30-Palma-Africana.pdf (consultado sep. 16-13).

ÁLVAREZ, Astrid (2009). Efectos del monocultivo de la palma de aceite en los medios de vida de las comunidades campesinas El caso de Simití Sur de Bolívar. Trabajo de grado de Maestría, Pontificia Universidad Javeriana, Maestría en Desarrollo Rural, 138p. (En línea) Disponible en http://www.javeriana.edu.co/biblos/ tesis/eambientales/tesis25.pdf (consultado sep. 12-13).

AVELLANEDA, Fredy (2010). Producción y caracterización de biodiesel de palma y aceite reciclado mediante un proceso batch y un proceso continuo con un reactor helicoidal. Tesis doctoral. Universitat Rovira I Virgili, Departament d'Enginyeria Química, 244p. (En línea) Disponible en http://www.tdx.cat/bitstream/ handle /10803/8588/Tesi.pdf?sequence=1 (consultado sep. 18-13). 
BALDOVINO, Renato (2011). Caracterización de tres modelos asociativos del cultivo de palma de aceite en el Municipio de Sabana de Torres Departamento de Santander. Trabajo de grado de Maestría, Pontificia Universidad Javeriana, Maestría en Desarrollo Rural, 112p. (En línea) Disponible en http://repository.javeriana.edu.co/bitstream/10554/1553/1/GuevarasyRuralesRenatoBaldovino2011.pdf (consultado sep. 10-13).

RODRÍGUEZ, Manuel; VAN HOFF, Bart (2003). El desempeño ambiental del sector palmicultor colombiano: una década de avances y un futuro promisorio. Palmas, Vol. 24, No. 3, pp. 69-86 (En línea) Disponible en http://www.manuelrodriguezbecerra.com/bajar/desenpeno_palmicultor.pdf (consultado sep. 17-13).

CARRERE, Ricardo (2001). El amargo fruto de la palma aceitera despojo y deforestación. Movimiento Mundial por los Bosques Tropicales, Novib, Sociedad Sueca para la Conservación de la Naturaleza. RelUita, 80p. (En línea) Disponible en http://www.wrm.org.uy/plantaciones/material/amar go_fruto.pdf (consultado sep. 13-13).

CARRERE, Ricardo (2006). Palma aceitera de la cosmética al biodiesel. Movimiento Mundial por los Bosques Tropicales, 128p. (En línea) Disponible en: http://www.wrm.org.uy/plantaciones/material/PalmaAceitera2. pdf (consultado sep. 10-13).

CARVAJAL, Leonardo; DE FRANCESCO, Hans (2012). Medio ambiente, recursos hídricos, y calentamiento global: significados y alcances en política exterior colombiana. En: ¿Colombia una potencia en desarrollo? Escenarios y desafíos para su política exterior, Konrad Adenauer Stiftung, Editor Stefan Jost, pp. 469-518 (En línea) Disponible en http://www.kas.de/wf/doc/6989-1442-4-30.pdf (consultado sep. 15-13).

CASTIBLANCO, Carmenza; HORTÚA, Sonia (2012). El paradigma energético de los biocombustibles: panorama mundial y el caso colombiano. Gestión y Ambiente, Vol. 15, No. 3, diciembre, pp. 5-25. (En línea) Disponible en http://www.redalyc.org/pdf/1694/169424893001.pdf (consultado sep. 24-13).

CASTRILLÓN, Nicolás (2008). Informe sobre desplazamiento forzado y problemática agraria. Asociación Campesina de Antioquia. El Ágora USB, Vol. 8, No. 2, pp. 241-474. (En línea) Disponible en http://web. usbmed.edu.co/usbmed/elagora/htm/v8nro2/documentospdf/catedra_abierta/Desplazamiento_y_ problem\%C3\%A1tica_Agraria_en_Colombia.pdf (consultado sep. 16-13).

CORTÉS, Raúl; MORENO, Daniela; POVEDA, Angie (2012). Análisis del impacto de la política de biocombustibles en la producción de aceite de palma y la estabilización del precio interno en Colombia. Revista Civilizar de Empresa y Economía, Vol. 3, No. 5, enero, pp. 81- 97. (En línea) Disponible en http://www. usergioarboleda.edu.co/civilizar/economia/edicion-5/N.5\%20ART.4.pdf (consultado sep. 25-13).

COVIELLO, Manlio; GÓMEZ, José; RAZO, Carlos; RODRÍGUEZ, Adrián (2008). Biocombustibles líquidos para transporte en América Latina y el Caribe. CEPAL, Colección Documentos de Proyectos. Santiago de Chile: Naciones Unidas, 183p. (En línea) Disponible en http://www.eclac.org/publicaciones/xml/7/36417/ Icw203e.pdf (consultado sep. 18-13).

CUESTA, Teófilo (2009). Evaluación interdimensional de impactos ambientales sobre la dimensión física ocasionados por cultivos de palma aceitera y la ganadería extensiva en la selva húmeda tropical del Bajo Atrato. Revista Gestión y Ambiente, Vol. 12, No. 3, agosto-diciembre, pp. 37-48 (En línea) Disponible en http://www.revistas.unal.edu.co/index.php/gestion/article/download/25319/25825 (consultado sep. 28-13).

FEDEPALMA (2012). Informe de gestión. Federación Nacional de Cultivadores de Palma de Aceite 2012, 240p. (En línea) Disponible en: publicaciones.fedepalma. org/index.php/labfedepalma/article/.../1062 (consultado oct. 1-13) (consultado ago. 2-13).

FIAN INTERNACIONAL (2009). Resultados de la Misión Internacional de Verificación sobre los impactos de los agrocombustibles en Colombia. Informe Regional Magdalena Medio, 47p. (En línea) Disponible en http://retornoalaspavas.files.wordpress.com/2011/02/informe-fian-palma-en-el-magadalena-medio. pdf (consultado sep. 23-13).

GARCÍA, Helena; CALDERÓN, Laura (2012). Evaluación de la política de biocombustibles en Colombia. Fedesarrollo, 154p. (En línea) Disponible en http://www.fedesarrollo.org.co/wp-content/uploads/2011/08/ Evaluaci\%C3\%B3n-de-la-pol\%C3\%ADtica-de-Biocombustibles-en-Colombia.pdf(consultado sep. 23-13). 
GARCÍA, Paola (2011). La paz perdida. Territorios colectivos, palma africana y conflicto armado en el Pacifico colombiano. Tesis Doctoral. Flacso, México, 303p. (En línea) Disponible en http://www.flacso.edu. mx/biblioiberoamericana/TEXT/DOCCS_VII_promocion_2008-2011/Garcia_P.pdf(consultado sep. 14-13).

GARCÍA, Rafael; NÚÑEZ, Adriana; RAMÍREZ, Tatiana; JAIMES, Sonia (2013). Caracterización de la fase UPSTREAM de la cadena de valor y abastecimiento de la agroindustria de la palma de aceite en Colombia. Dyna, año 80, No 179, junio, Medellín, pp. 79-89. (En línea) Disponible en http://dyna. unalmed.edu.co/ ediciones/179/articulos/v80n179a09/v80n179a09.pdf (consultado de sep. 22. 13).

GOEBERTUS, Juanita (2008). Palma de aceite y desplazamiento forzado en la zona bananera: trayectorias entre recursos naturales y conflictos. Colombia Internacional, No 67, enero - junio, pp. 152-175. (En línea) Disponible en http://colombiainternacional.uniandes.edu.co/datos/pdf/descargar.php?f=./data/ Revista_No_67/08_Nuevas_2.pdf (consultado sep. 23-13).

HURTADO, Mónica; HERNÁNDEZ, Giovanni (2010). Perfil local y agroindustria palmera: explorando el caso de San Alberto y San Martín (Cesar). Cuadernos de Desarrollo Rural, vol 7, No 65, pp. 127-149. (En línea) Disponible en http://www.scielo.org.co/pdf/cudr/v7n65/v7n65a07.pdf (consultado oct. 4-13).

HURTADO, Mónica; PEREIRA, Catherine (2011). Legitimidad empresarial, conflicto de tierras y producción palmera en Colombia. Revista de Relaciones Internacionales, Estrategia y Seguridad, vol 6, No 2, juliodiciembre, pp. 91-110. (En línea) Disponible en http://www.umng.edu.co/documents/63968/76123/4. pdf (consultado oct. 3-13).

INDUPALMA (2011). Informe de Sostenibilidad 2010 - 2011. INDUPALMA, 118p. (En línea). Disponible en http://www.indupalma.com/sites/default/files/file_attach/Informe\%20Sostenibilidad\%20INDUPALMA\%202010-2011.pdf (consultado oct. 10-13).

INFANTE, A. Y TOBÓN, S. (2010). Bioenergía para el desarrollo sostenible. Políticas Públicas sobre Biocombustibles y su relación con la seguridad alimentaria en Colombia. Ministerio de Asuntos Exteriores y de Cooperación- AECID - FAO, 120p. (En línea) Disponible en http://www.eldulcesabordel progreso. com/uploads/attachment/fa2dc0d0bfff6b7d27ba1f6581b139ba8eb6cb30.pdf (consultado oct. 8-13).

KONRAD ADENAUER STIFTUNG (2008). Expansión de la palma. En: Kas papers, No. 2, noviembre, pp. 2-21. (En línea) Disponible en http://www.kas.de/wf/doc/kas_15037-544-4-30.pdf (consultado sep. 24- 13).

LA ROTTA, Gustavo (2010). Efectos sociales del cultivo de palma de aceite: condiciones laborales, seguridad social, y educación en los trabajadores palmeros de Cumaral. Trabajo de grado de Maestría. Pontificia Universidad Javeriana. Maestría en Desarrollo Rural, 139p. (En línea) Disponible en http://repository. javeriana.edu.co/bitstream/10554/701/1/pol137.pdf (consultado sep. 15-13).

LONDOÑO, Patricia; PINEDA, Liliana (2008). Los negocios inclusivos en Colombia. Alianza para los negocios inclusivos, Servicio Holandés de Cooperación al Desarrollo (SNV), Consejo Empresarial Colombiano para el Desarrollo Sostenible, CECODES, 46p. (En línea) Disponible en http://www.snvworld.org/sites/ www.snvworld.org/files/publications/los_negocios_inclusivos_en_colombia.pdf (consultado sep. 14-13).

LÓPEZ, Angie (2010). Estimación de conflictos de uso de la tierra por dinámica de cultivos de palma africana, usando sensores remotos. Caso: Departamento del Cesar. Trabajo de grado de Maestría, Universidad Nacional de Colombia, Escuela de Geociencias y Medio Ambiente, Medellín, 78p. (En línea). Disponible en http://www.bdigital.unal.edu.co/1886/1/43254215.20101.pdf (consultado sep. 15-13).

LÓPEZ, Nelson (2000). La palma de aceite: un caso exitoso de desarrollo empresarial en Colombia. Palmas, vol 21, No Especial, tomo 2, pp. 132-141. (En línea) Disponible en: http://portal.fedepalma.org//responsabilidad_social palma_aceite_caso_exitoso.pdf (consultado sep. 25-13).

MARTíNEZ, Héctor; ESPINAL, Carlos; SALAZAR, Marcela; BARRIOS, Camilo (2005). La cadena de las oleaginosas en Colombia. Una mirada global de su estructura y dinámica 1995-2005. Ministerio de Agricultura y Desarrollo Rural, Observatorio Agrocadenas Colombia, documento de trabajo No. 62, 32p. (En línea). Disponible en: http://www.agronet.gov.co/www/docs_agronet/20051 12162648_caracterizacion_oleaginosas.pdf (consultado sep. 15-13). 
MINISTERIO DE MEDIO AMBIENTE, VIVIENDA Y DESARROLLO TERRITORIAL Y FEDEPALMA (2011). Guía ambiental de la agroindustria de la palma de aceite en Colombia. Versión para consulta, 86p. (En línea) Disponible en http://portal.fedepalma.org//documen/2011/Guia_Ambiental.pdf(consultado sep. 22-13).

MINGORANCE, Fidel; MINELLI, Flaminia; LE DU, Hélène (2004). El cultivo de la palma africana en el Chocó. Legalidad ambiental, territorial y derechos humanos. Human Rights Everywhere y Diócesis de Quibdó, primera edición, 180p. (En línea) Disponible en http://www.raulzelik.net/images/rztextarchiv/uniseminare/ Palma_africana_Choco.pdf (consultado sep. 24-13).

MOSQUERA, Mauricio; BERNAL, Paloma; SILVA, Álvaro (2009). Agenda prospectiva de investigación y desarrollo para la cadena de oleaginosas, grasas y aceites en Colombia con énfasis en oleína roja. Ministerio de Agricultura y Desarrollo Rural, Universidad Nacional de Colombia, Cenipalma, Fedepalma, 184p (En línea) Disponible en http://www.agronet.gov.co/www/docs_agronet/2009424103533_OLEINA.pdf (consultado sep. 10.13).

NACIONES UNIDAS (1987). Asamblea General. Cuadragésimo segundo período de sesiones. Anexo Informe de la Comisión Mundial sobre el Medio Ambiente y el Desarrollo "Nuestro futuro común", 416p. (En línea) Disponible en http://es.scribd.com/doc/178958413/Informe-Bruntdland-Nuestro-futuro-comun (consultado oct. 3-13).

NIEVES, María (1998). Género, medio ambiente y sustentabilidad del desarrollo. Comisión Económica para América Latina - CEPAL -, Serie Mujer y Desarrollo, Santiago de Chile: Naciones Unidas, 51p. (En línea) Disponible en http://www.eclac.org/publicaciones/xml/3/4343/lcl1144e.pdf (consultado oct. 4-13).

OCAMPO, Sebastián (2009). Agroindustria y conflicto armado: el caso de la palma de aceite. Colombia Internacional, 70, julio a diciembre, pp. 169 - 190. (En línea). Disponible en: http://colombiainternacional. uniandes.edu.co/view.php/487/ view.php (consultado sep. 26-13).

OCHOA, Juan; CHÁVEZ, Ernesto (2011). Evaluación de la sostenibilidad en los cultivos de palma africana en el Departamento del Meta. Contribuciones a las Ciencias Sociales, septiembre, 6p. (En línea) Disponible en http://www. eumed.net/rev/cccss/13/oach.html (consultado sep. 20-13).

OLIVERA, Mauricio; ESCOBAR, Diego; ROJAS, Norbeto; MORENO, Julián; QUINTERO, Claudia; TIBOCHA, Ana (2011). Caracterización del empleo en el sector palmicultor colombiano. Cuadernos de Fedesarrollo, No 37, 113p. (En línea) Disponible en http://www.fedesarrollo.org.co/wp-content/uploads/2011/08/ Cuaderno-No.-37.pdf (consultado sep. 13-13).

PÉREZ, Mario (2010). Dinámica del sector palmero en Colombia y la región del Sur de Bolívar: Análisis de sus conflictos ambientales. Cali, Universidad del Valle, Instituto Cinara, 18p. (En línea) Disponible en http:// seminarioambienteycultura.bligoo.com.co/media/users/8/433012/files/34823/Perez_Mario_2010_b_. pdf (consultado sep. 21-13).

PERFETTI, Mauricio; RICARDO, Herman; ORTIZ, Oscar; ZÁRATE, Catalina; MUÑOZ, Jorge; ARANGO, Pablo (2003). Impactos socioeconómicos de la agroindustria de palma de aceite en Colombia. Centro de Estudios Regionales Cafeteros y Empresariales, 120p. (En línea) Disponible en http://portal.fedepalma.org// responsabilidad_social/Informe_CRECE.pdf (consultado sep. 2-13).

PRETI, Alessandro; GONZÁLEZ, Olga; VILLEGAS, Astrid (2010). Los Montes de María: análisis de la conflictividad. P.N.U.D. Colombia, ASDI, 60p. (En línea) Disponible en http://www.undp.org/content/dam/ undp/documents/projects/COL/00058220/Analisis\%20conflcitividad\%20Montes\%20de\%20Maria\%20 PDF.pdf (consultado sep. 25-13).

PRETI, Alessandro; GONZÁLEZ, Olga; VILLEGAS, Astrid (2010). Cesar, análisis de la conflictividad. PNUD Colombia, Área de paz desarrollo y reconciliación - ASDI, 64p. (En línea) Disponible en http://www.undp. org/content/dam/undp/documents/projects/COL/00058220/Analisis\%20Cesar\%20Definitivo\%20PDF. pdf (consultado oct. 3-13).

RAMÍREZ, Rosa (2012). Diagnóstico sobre la situación de los trabajadores de la agroindustria de la palma en el Magdalena Medio y el Meta. Informe final Proyecto FOS-FENSUAGRO, 128p. (En línea) Disponible en: http://comunicandes.org/pdf/informe_palmero_colombia.pdf (consultado sep. 10-13). 
RIVAS, Angela; RETTBERG, Angelika; ARBOLEDA, Juliana; CAJIAO, Andrés (2011). Sector privado y desplazamiento forzado interno (DFI) en Colombia. Fundación Ideas para la Paz y Universidad de los Andes, 50p. (En línea) Disponible en: http://www.ideaspaz.org/portal/images/desplazamientoweb.pdf (consultado sept. 3-13).

RODRÍGUEZ, Manuel; RUDAS, Guillermo; ANDRADE, Germán; URIBE, Eduardo; CASTRO, Luis; WILLS, Eduardo; DURÁN, Armando (2009). La mejor Orinoquía que podemos construir. Elementos para la sostenibilidad ambiental del desarrollo, Corporinoquia - Universidad de los Andes - Foro Nacional Ambiental - Friedrich Ebert Stiftung, 144p. (En línea) Disponible en http://library.fes.de/pdf-files/bueros/kolumbien/08358.pdf (consultado sep. 16 -13).

ROJAS, Cornelio (2003). El desarrollo sustentable: un nuevo paradigma en la Administración Pública. Instituto Nacional de Administración Pública A.C, México, primera edición, 270p. (En línea) Disponible en http://www.inap.org.mx/portal/images/RAP/29\%20el\%20desarrollo\%20sustentable\%20nuevo\%20 paradigma.pdf (consultado ago. 15-13).

SÁNCHEZ, Liliana (2009). El empleo en el cultivo de palma africana. Contraloría General de la República. Contraloría Delegada Sector Agropecuario, Dirección de Estudios Sectoriales, 8p. (En línea). http://186.116.129.19/c/document_library/get_file?\&folderld=44944020\&name=DLFE-34932.pdf recuperado 2 de agosto de 2013 (consultado ago. 8-13).

SEEBOLT, Sandra; SALINAS, Yamile (2010). Responsabilidad y sostenibilidad de la industria de palma ¿Son factibles los criterios de RSPO en Colombia? Oxfam- Novib- Indepaz, 76p. (En línea) Disponible en http:// ediciones.indepaz.org.co/2012/02/publicacion-9/ (consultado ago. 15-13).

SILVA, Álvaro; MARTíNEZ, Gerardo (2009). Plan nacional del manejo de la pudrición del cogollo. Fedepalma-Cenipalma. Palmas, Vol. 30, No. 3, pp. 97-121 (En línea). Disponible en: http://portal.fedepalma.org// documen/2009/plan_manejo_PC.pdf (consultado ago. 7-13).

SOLER, Juan; LEÓN, Daniel (2009). Impactos ambientales de la expansión de palma aceitera en el Magdalena Medio, hablan los pobladores. Asociación campesina de cacaoteros de Buenos Aires -Programa de Desarrollo y Paz del Magdalena Medio- Proyecto Regional de Tierras, 71p. (En línea) Disponible en http://prensarural.org/spip/IMG/pdf/Informe_de_Impactos_Ambientales_Version_FINAL._1_.pdf(consultado oct. 10-13).

SUPERINTENDENCIA DE INDUSTRIA Y COMERCIO (2010). Estudios de Mercado. Estudio de la agroindustria de la palma africana en Colombia (2010- 2011), 25p. (En línea) Disponible en: http://www.sic.gov.co/ recursos_user/documentos/publicaciones/pdf/PalmaAfricana2012.pdf (consultado sep. 23-13).

TOBASURA, Isaías (2009). De campesinos a empresarios: la retórica neoliberal de la política agraria en Colombia. Agronomía, 17 (1), pp. 21-34, (en línea). Disponible en http://agronomia.ucaldas.edu.co/ downloads/Agronomia17\%281\%29_4.pdf (consultado ago. 30-13).

VIDAL, Carlos; VILLARROEL, Ingreidis (2011). Alianzas estratégicas para el cultivo de palma de aceite. Una metodología de recuperación e integración agroindustrial en María La Baja, Departamento de Bolívar Colombia. Tecnología, Gerencia y Educación, Vol. 12, No. 24, diciembre, pp. 145 - 159. (En línea) Disponible en http://revista.iutet.edu.ve/V12_24_2.pdf (consultado sep. 11-13).

WILCHES, Angela (2011). Aspectos bioéticos en la producción de combustibles en Colombia. Tesis doctoral, Universidad El Bosque, Doctorado en Bioética, 153p. (En línea) Disponible en: http://www.bioeticaunbosque.edu.co/ Programas/pdftesisdoctorales/angelawilches.pdf (consultado sep. 14-13). 